\title{
Nonclassical light revealed by the joint statistics of simultaneous measurements
}

\author{
Alfredo LUIS* \\ ${ }^{1}$ Departamento de Óptica, Facultad de Ciencias Físicas, Universidad Complutense, 28040 Madrid, Spain \\ *Corresponding author: alluis@fis.ucm.es
}

Compiled March 5, 2016

Nonclassicality cannot be a single-observable property since the statistics of any quantum observable is compatible with classical physics. We develop a general procedure to reveal nonclassical behavior of light states from the joint statistics arising in the practical measurement of multiple observables. Besides embracing previous approaches, this protocol can disclose nonclassical features for standard examples of classical-like behavior, such as $S U(2)$ and Glauber coherent states. When combined with other criteria this would imply that every light state is nonclassical. @ 2016 Optical Society of America

OCIS codes: (270.0270) Quantum optics; (270.5290) Photon statistics; (030.6600) Statistical optics (000.1600) Classical and quantum physics;

\section{http://dx.doi.org/10.1364/ao.XX.XXXXXX}

Nonclassicality cannot be a single-observable property, since within classical physics it is always possible to reproduce exactly the statistics of any quantum observable. For example, we can consider the observable of photon number, that means field energy in units of the one-photon energy. In classical electromagnetism, a wave can have any value of the energy with zero uncertainty. This includes any integer number of times the energy of a photon, mimicking the energy statistics of a Fock state. Moreover, in classical optics waves can be generated with random amplitudes, with probabilities reproducing exactly the number distribution of any quantum field state. Actually, a key feature of the quantum versus classical relationship is quite the opposite: there are classical statistics that cannot be reproduced by quantum states, such as perfectly defined field energy different from integer values of the photon energy.

Thus, nonclassical effects must be found when addressing the joint statistics of multiple observables, specially if they are incompatible [1]. In the most general case joint measurements require the coupling of the system with auxiliary degrees of freedom. This means that the measurement must be followed by some kind of data analysis or inversion procedures to extract the information of the system variables from the observed statistics in the enlarged space [2]. We show that in classical physics the result of the inversion is always a true joint probability distribu- tion of system variables. However, in quantum physics this is not always the case and the result of the inversion can be incompatible with classical statistics. Parallels can be drawn with the construction joint probability distributions via the inversion of moments [3].

This is a very general program that includes as particular cases classic demonstrations of the nonclassical behavior of some light states, such as coincidence detection and quantum tomography [4]. Besides, this approach discloses nonclassical properties for light states that otherwise are universally considered as classical light. Furthermore, when combined with other nonclassical criteria it would imply that every light state is nonclassical.

We consider the simultaneous measurement of two compatible observables $\tilde{X}$ and $\tilde{Y}$, with outcomes $x$ and $y$, respectively, and an joint probability $\tilde{p}_{X, Y}(x, y)$. The corresponding marginal distributions are

$$
\tilde{p}_{X}(x)=\sum_{y} \tilde{p}_{X, Y}(x, y), \quad \tilde{p}_{Y}(y)=\sum_{x} \tilde{p}_{X, Y}(x, y),
$$

where we are assuming a discrete range for $x$ and $y$ without loss of generality. We consider that these marginals provide information about two system observables $X$ and $Y$, respectively, that may be compatible or not. We assume that the information about $X$ and $Y$ contained in $\tilde{p}_{X, Y}$ is complete, so that the distributions $p_{Z}(z)$ for $Z=X, Y$ and $z=x, y$ can be retrieved from the observed marginals $\tilde{p}_{Z}(z)$ above in the form

$$
p_{Z}(z)=\sum_{z^{\prime}} \mu_{Z}\left(z, z^{\prime}\right) \tilde{p}_{Z}\left(z^{\prime}\right)
$$

where $\mu_{Z}$ is a matrix with matrix elements $\mu_{Z}\left(z, z^{\prime}\right)$ completely known as far as we know the measurement being performed. These are the non ideal invertible measurements introduced in Ref. [2].

The key idea is to extend this inversion from the marginals to the complete joint distribution in the form

$$
p_{X, Y}(x, y)=\sum_{x^{\prime}, y^{\prime}} \mu_{X}\left(x, x^{\prime}\right) \mu_{Y}\left(y, y^{\prime}\right) \tilde{p}_{X, Y}\left(x^{\prime}, y^{\prime}\right)
$$

or in matrix form

$$
p_{X, Y}=\mu_{X} \tilde{p}_{X, Y} \mu_{Y}^{t}
$$

where the superscript $t$ denotes matrix transposition.

Let us show that in classical physics the inversion procedure (3) always leads to a bona fide distribution $p_{X, Y}(x, y)$. Classically 
the state of the system can be completely described by a legitimate probability distribution $P(\lambda)$, where $\lambda$ is a point in the corresponding phase space. So the observed joint statistics can be always expressed in the form

$$
\tilde{p}_{X, Y}(x, y)=\int \mathrm{d} \lambda P(\lambda) \tilde{X}(x \mid \lambda) \tilde{Y}(y \mid \lambda)
$$

where $\tilde{Z}(z \mid \lambda)$ are the conditional probabilities that the observable $\tilde{Z}$ takes the value $z$ when the system state is $\lambda$. Applying Eq. (2)

$$
Z(z \mid \lambda)=\sum_{z^{\prime}} \mu_{Z}\left(z, z^{\prime}\right) \tilde{Z}\left(z^{\prime} \mid \lambda\right) .
$$

we readily get from Eqs. (3) and (6)

$$
p_{X, Y}(x, y)=\int \mathrm{d} \lambda P(\lambda) X(x \mid \lambda) Y(y \mid \lambda)
$$

which is from the actual joint distribution for $X$ and $Y$. Therefore, in the classical domain the inversion works equally well for the joint distribution as for the marginals so the lack of positivity or any other pathology of the retrieved joint distribution $p_{X, Y}(x, y)$ is a signature of nonclassical behavior.

The simplest example illustrating this approach deals with the statistics of two complementary observables in a qubit. In quantum optics the qubit can be implemented as the polarization of a single photon in a two-mode electromagnetic field. We consider two dichotomic observables with outcomes $x, y= \pm 1$, describing for example linear polarization (horizontal versus vertical) and circular polarization (dextro versus levo), respectively, and represented as quantum operators by the Pauli matrices $X=\sigma_{x}$ and $Y=\sigma_{y}$. The most general photon state is given by the density matrix

$$
\rho=\frac{1}{2}\left(\sigma_{0}+\boldsymbol{s} \cdot \boldsymbol{\sigma}\right)
$$

where $\sigma_{0}$ is the identity, $\sigma$ are the three Pauli matrices, and $s$ is a three-dimensional real vector with $|\boldsymbol{s}| \leq 1$, these are the Stokes parameters. So the system space for the qubit is the unit Bloch sphere that in our example coincides with the Poincaré sphere.

The exact statistics are

$$
p_{X}(x)=\frac{1}{2}\left(1+x s_{x}\right), \quad p_{Y}(y)=\frac{1}{2}\left(1+y s_{y}\right) .
$$

A joint measurement of these observables can be carried out as described in detail for example in Refs. [5] and [6], leading to the joint statistics

$$
\tilde{p}_{X, Y}(x, y)=\frac{1}{4}\left(1+x s_{x} \cos \phi+y s_{y} \sin \phi\right),
$$

where $\phi$ is an apparatus parameter. The corresponding marginals

$$
\tilde{p}_{X}(x)=\frac{1}{2}\left(1+x s_{x} \cos \phi\right), \quad \tilde{p}_{Y}(y)=\frac{1}{2}\left(1+y s_{y} \sin \phi\right),
$$

which can be easily inverted in the form (2) with

$$
\mu_{Z}\left(z, z^{\prime}\right)=\frac{1}{2}\left(1+\frac{z z^{\prime}}{\kappa_{Z}}\right),
$$

where $\kappa_{X}=\cos \phi$ and $\kappa_{Y}=\sin \phi$. Finally, the inversion (4) leads to

$$
p_{X, Y}(x, y)=\frac{1}{4}\left(1+x s_{x}+y s_{y}\right) \text {. }
$$

Clearly $p_{X, Y}(x, y)$ can take negative values if $|\boldsymbol{s}|>1 / \sqrt{2}$. Relying on the spherical symmetry we may say that any state with $|\boldsymbol{s}|>1 / \sqrt{2}$ is actually nonclassical after a suitable choice of the $X, Y$ observables. This represents the $65 \%$ of the volume of the Bloch sphere. This is quite relevant since it is often argued that all $1 / 2$ spin states are classical since all them have a wellbehaved Glauber-Sudarshan SU(2) P-function [7]. Actually, the more nonclassical states are the $\mathrm{SU}(2)$ coherent states since $|\boldsymbol{s}|=$ 1. Previous reports on the non classicality of these states can be found in Ref. [8].

In the next example the system is an electromagnetic field mode in some state $\rho$. This is mixed with a Glauber coherent state of mean photon number $\bar{n}$ at a lossless $50 \%$ beam splitter and the number of photons $n_{1}$ and $n_{2}$ are registered at the two output ports with joint statistics $\tilde{p}^{\prime}\left(n_{1}, n_{2}\right)$. The variables of interest for this example are not $n_{1,2}$, but the total number $N=n_{1}+n_{2}$ and the normalized number difference $m=\left(n_{1}-n_{2}\right) /\left(n_{1}+n_{2}\right)$. The observed joint distribution for these variables is

$$
\tilde{p}(N, m)=\tilde{p}^{\prime}\left(n_{1}=N \frac{1+m}{2}, n_{2}=N \frac{1-m}{2}\right),
$$

where $N=0,1, \ldots, \infty$, while the range for $m$ is the union of all the ranges allowed for each $N$, this is $m=-1,-1+2 / N, \ldots, 1$, and we consider just $m=0$ for $N=0$. For simplicity we drop the subscripts in $p$ and $\tilde{p}$ since there is no risk of confusion.

The marginal statistics for the total number $\tilde{p}(N)$

$$
\tilde{p}(N)=\sum_{m \in M} \tilde{p}(N, m)=e^{-\bar{n}} \sum_{n=0}^{N} \frac{\bar{n}^{N-n}}{(N-n) !} p(n),
$$

can be regarded as providing information about the photonnumber distribution $p(n)=\langle n|\rho| n\rangle$ of the system state $\rho$. The corresponding data inversion is of the form

$$
p(n)=e^{\bar{n}} \sum_{N=0}^{n} \frac{(-\bar{n})^{n-N}}{(n-N) !} \tilde{p}(N) .
$$

On the other hand, the marginal distribution $\tilde{p}(m)$ represents relative-phase statistics in terms of the typical visibility of the interference at the beam splitter. More specifically, $\tilde{p}(m)$ can be related to well-known approaches to the cosine of the relative phase introduced via homodyne detection [9]. Because of this, and for the sake of simplicity we consider that $\tilde{p}(m)$ requires no data inversion. This is consistent with the understanding that there is no operator for the single-mode phase which is more properly represented by positive operator measures [10,11].

Therefore, applying the inversion (16) to the complete joint distribution we get

$$
p(n, m)=e^{\bar{n}} \sum_{N=0}^{n} \frac{(-\bar{n})^{n-N}}{(n-N) !} \tilde{p}(N, m) .
$$

Nonclassical behavior holds for example when considering the particular values $n=1$ and $m=0$. Note that $N$ is the sum of the number photons recorded in both detectors, so that this example deals with the detection either of no photons, or at most a single photon in only one detector. Taking into account that $m=0$ is not included for $N=1$

$$
p(1,0)=-\bar{n} e^{\bar{n}} \tilde{p}(0,0)=-\bar{n} p(0) .
$$

Therefore, whenever $p(0) \neq 0$ and $\bar{n} \neq 0$ we have $p(1,0)<0$. In particular this discloses non classical properties for the Glauber 
coherent states and even for thermal states, which are widely assumed as classical-like states. The most simple example is the vacuum state which is both thermal and coherent, with maximum $p(0)=1$. The fact the coherent states display nonclassical behavior for number-phase variables was already noticed in Refs. [12].

The main objective of this work is a proof of principle of the method so ideal conditions have been assumed throughout. Naturally, its practical implementation will face the effects of typical experimental imperfections [13]. Let us briefly show that they present no any insuperable obstacle to the observation of nonclassical results in the cases presented above. To this end practical imperfections are exemplified by finite quantum efficiency $\eta$, and finite sampling.

Concerning the one-photon polarization example, for definiteness let us consider the case $s_{x}=s_{y}=|\boldsymbol{s}| / \sqrt{2}$ with $\phi=\pi / 4$. Since we have only one photon, the effect of the finite quantum efficiency is that only a fraction $\eta$ of the $N$ repetitions of the measurement will actually contribute to the readouts. These actually recorded photons will tend to the observed statistics

$$
\tilde{p}_{X, Y}( \pm 1, \pm 1)=\frac{1}{4}(1 \pm|s|), \quad \tilde{p}_{X, Y}( \pm 1, \mp 1)=\frac{1}{4} .
$$

The inversion procedure leading to

$$
p_{X, Y}(-1,-1)=\frac{1}{4}(1-\sqrt{2}|\mathbf{s}|)
$$

that takes negative values for $|\boldsymbol{s}|>1 / \sqrt{2}$. The finite quantum efficiency will manifest in the uncertainty of the observed statistics, that will follow a binomial distribution

$$
\Delta^{2} \tilde{p}_{X, Y}(x, y)=\frac{\tilde{p}_{X, Y}(x, y)\left[1-\tilde{p}_{X, Y}(x, y)\right]}{\eta N},
$$

that depends on the number of effective runs $\eta N$. A simple propagation of errors leads to

$$
\Delta^{2} p_{X, Y}(-1,-1)=\frac{54-24 \sqrt{2}|\boldsymbol{s}|-17|\boldsymbol{s}|^{2}}{128 \eta N} .
$$

With this we can easily examine the statistical significance of the negativity of $p_{X, Y}(-1,-1)$. We can ask for the number of repetitions $N$ so that $p_{X, Y}(-1,-1)$ is more than $v$ standards deviations below zero, for example for $|\boldsymbol{s}|=1$,

$$
\left|p_{X, Y}(-1,-1)\right| \geq v \Delta p_{X, Y}(-1,-1) \longrightarrow N \geq 2.2 \frac{v^{2}}{\eta} .
$$

For $v=10$ and a rather low quantum efficiency $\eta=0.1$ this condition is satisfied when $N \geq 2.2 \times 10^{3}$, which is a rather accessible number of repetitions.

Regarding the number-phase example in Eq. (18), and in the same spirit above we get that

$$
p(1,0)=-\eta \bar{n} e^{\eta \bar{n}} \tilde{p}(0,0), \quad \tilde{p}(0,0)=e^{-\eta \bar{n}},
$$

with uncertainty

$$
\Delta p(1,0)=\eta \bar{n} e^{\eta \bar{n}} \Delta \tilde{p}(0,0)=\eta \bar{n} \sqrt{\frac{e^{\eta \bar{n}}-1}{N}} .
$$

Thus imposing that $p(1,0)$ is more than $v$ standard deviations below zero

$$
|p(1,0)| \geq v \Delta p(1,0) \longrightarrow N \geq v^{2}\left(e^{\eta \bar{n}}-1\right) .
$$

For example, for $\bar{n}=10, \eta=0.1$ and $v=10$ this means $N \geq$ $1.7 \times 10^{2}$, which is again a very accessible result.

Finally we comment on the main points addressed above. We have addressed an universal protocol to disclose nonclassical behavior of light states via joint measurements of more than one observable. This includes previous criteria as particular cases and admits many other possibilities under one and the same framework.

In this regard, a current approach to mesuring nonclassicality of light consists of performing optical tomography and looking for nonclassical features of the Wigner distribution [14]. This can be regarded as a particular case of our approach, since when the measured observables are the field quadratures, the result of the inversion is the same Wigner function reconstructed by optical tomography, as shown in Ref. [2]. Nevertheless, this method goes beyond the reconstruction of standard phase-space distributions as demonstrated by the above example revealing nonclassical properties of Glauber coherent states.

After Eq. (5) we may say that the failure of the inversion procedure (3) holds because the observed statistics $\tilde{p}_{X, Y}(x, y)$ is not separable, this is that it cannot be expressed in the form

$$
\tilde{p}_{X, Y}(x, y)=\sum_{m} f_{m}(x) g_{m}(y),
$$

for some positive functions $f_{m}$ and $g_{m}$. For example, if we try to express $\tilde{p}_{X, Y}(x, y)$ in Eq. (10) in this factorized form (27) we get

$$
\tilde{p}_{X, Y}(x, y)=\frac{1}{2}\left[\tilde{p}_{X}(x)+\tilde{p}_{Y}(y)\right]-\frac{1}{4},
$$

so this is never separable for $|\boldsymbol{s}| \neq 0$.

This lack of separability may have two causes: either there is no well-behaved $P(\lambda)$, or the factorization $X(x \mid \lambda) Y(y \mid \lambda)$ fails. This points to two sources of nonclassical features: the observed state and the observing procedure. Let us elaborate in this. When a measurement is made on some system state $|\psi\rangle$ resulting the outcome $a$, we may say that the system is found in the state corresponding to the outcome, say $|a\rangle$. For most measuring schemes such states $|a\rangle$ are actually nonclassical, say number states for photodetection or squeezed states for quadrature measurements in homodyne detection [15]. The intriguing point is that the observed statistics emerges from the concurrence of the observed state and the apparatus states via the Born rule in a completely symmetric way $|\langle a \mid \psi\rangle|^{2}$ [16]. This raises the question of whether nonclassicality is a property of the apparatus states $|a\rangle$ or a property of the state being measured $|\psi\rangle$. Up to now the attention has focused on the system state, and the observation was merely an instrument. We hope that the analysis developed in this work may shed light on this point since the standard approach may be overlooking many interesting results conveyed by nonclassical measurements [17].

This might be the case in the above phase-number example. There, the nonclassicality may be ascribed to same kind of effective entanglement between the $N$ and $m$ variables, since the allowed values for $m$ may be different for different $N$. This $m-N$ entanglement is corroborated by most approaches to the quantum relative phase as a purely quantum effect [11]: in the classical case there is no relation whatsoever between the intensity and the cosine of the relative phase. In this regard, a deep relation between non classicality and entanglement has been put forward in Ref. [18].

Finally, let us note that the result in Eq. (18) is the dual of the nonclassical Lee criterion [19]. Such approach introduces a 
continuous parameter, referred to as nonclassical depth, to measure how nonclassical quantum states are. Roughly speaking, it means how many thermal photons must be incoherently added to a given state so its Glauber-Sudarshan P function becomes a classical-like distribution. A suitable theorem [20] demonstrates that if the probability of having zero photons vanishes, $p(0)=0$, then the nonclassical depth is maximum. This is quite the opposite of the result found here around Eq. (18). This means that the union of both criteria would imply that all light states of a one-mode field are nonclassical.

Funding. Projects from the Spanish Ministerio de Economía y Competitividad No. FIS2012-35583 and from the Comunidad Autónoma de Madrid research consortium QUITEMAD+ grant S2013/ICE-2801.

\section{REFERENCES}

1. J. S. Bell, Physics 1, 195 (1964); J. S. Bell, Rev. Mod. Phys. 38, 447 (1966); J. F. Clauser and M. A. Horne, Phys. Rev. D 10, 526 (1974); A. Fine, Phys. Rev. Lett. 48, 291 (1982); I. Pitowsky, Brit. J. Phil. Sci. 45, 95 (1994); T. Durt, Found. Phys. 27, 415 (1997); A. Rivas, http://arxiv.org/abs/1501.04929.

2. W. M. Muynck, Foundations of Quantum Mechanics, an Empiricist Approach, (Kluwer Academic Publishers, 2002); W. M. de Muynck and H. Martens, Phys. Rev. A 42, 5079 (1990); W. M. de Muynck, Phys. Lett. A 182, 201 (1993); W. M. de Muynck, J. Phys. A: Math. Gen. 31, 431 (1998).

3. H. S. Karthik, H. Katiyar, A. Shukla, T. S. Mahesh, A. R. Usha Devi, and A. K. Rajagopal, Phys. Rev. A 87, 052118 (2013).

4. L. Mandel and E. Wolf, Optical Coherence and Quantum Optics (Cambridge University Press, 1995); M. O. Scully and M. S. Zubairy, Quantum Optics (Cambridge University Press, 1997); D.-G. Welsch, W. Vogel, and T. Opatrný, in Progress in Optics, E. Wolf ed. (Elsevier Science, 1999) 39, 63.

5. A. Luis, Phys. Rev. A 84, 034101 (2011); A. Luis, http://arxiv.org/abs/1306.5211; A. Luis, G. M. Bosyk, and M. Portesi, Physica A 444, 905 (2016).

6. A. Luis, http://arxiv.org/abs/1506.07680.

7. F. T. Arecchi, E. Courtens, R. Gilmore, and H. Thomas, Phys. Rev. A 6, 2211 (1972); O. Giraud, P. Braun, and D. Braun, Phys. Rev. A 78, 042112 (2008); O. Giraud, P. Braun, and D. Braun, New J. Phys. 12, 063005 (2010).

8. D. Arsenović, N. Burić, and D. M. Davidović, Phys. Rev. A 60, 1797 (1999); A. Luis, Phys. Rev. A 73, 063806 (2006); M. V. Chokhova, and F. Y. Khalili, Phys. Rev. A 88, 023822 (2013).

9. P. Carruthers and M. M. Nieto, Rev. Mod. Phys. 40, 411 (1968); S. M. Barnett and D. T. Pegg, J. Phys. A 19, 3849 (1986); R. Lynch, J. Opt. Soc. Am. B 4, 1723 (1987); J. W. Noh, A. Fougères and L. Mandel, Phys. Rev. Lett. 67, 1426 (1991); J. W. Noh, A. Fougères and L. Mandel, Phys. Rev. A 45, 424 (1992); J. W. Noh, A. Fougères and L. Mandel, Phys. Rev. A 46, 2840 (1992); A. S. Shumovsky, Opt. Commun. 136, 219 (1997); A. Cives-Esclop, A. Luis, and L. L. Sánchez-Soto, Opt. Commun. 175, 153 (2000).

10. M. Grabowski, Int. J. Theor. Phys. 28, 1215 (1989); M. Grabowski, Rep. Math. Phys. 29, 377 (1991); J. Bergou and B.-G. Englert, Ann. Phys. (N. Y.) 209, 479 (1991); R. Lynch, Phys. Rep. 256, 367 (1995); V. Peřinová, A. Lukš and J. Peřina, Phase in Optics (World Scientific, 1998).

11. A. Luis and L. L. Sánchez-Soto, in Progress in Optics, E. Wolf ed. (Elsevier, 2000) 41, 421 (2000); A. Luis and L. L. Sánchez-Soto, Phys. Rev. A 48, 4702 (1993).

12. J. Vaccaro, Phys. Rev. A 52, 3474 (1995); A. Luis, Phys. Rev. A 73, 063806 (2006).

13. T. Kiss, U. Herzog, and U. Leonhardt, Phys. Rev. A 52, 2433 (1995).

14. K. Vogel and H. Risken, Phys. Rev. A 40, 2847 (1989); A. I. Lvovsky, H. Hansen, T. Aichele, O. Benson, J. Mlynek, and S. Schiller, Phys. Rev. Lett. 87, 050402 (2001).

15. N. G. Walker, J. Mod. Opt. 34, 15 (1987); O. Jedrkiewicz, R. Loudon, and J. Jeffers, Eur. Phys. J. D 39, 129 (2006).
16. In passing, this recalls the Goethe formulation of vision as the meeting of the inner and outer lights at the eye, as recalled by A. G. Zajonc, Am. J. Phys. 44, 327 (1976).

17. A. Rivas and A. Luis, Phys. Rev. A 79, 042105 (2009); A. Luis and A. Rivas, Phys. Scr. T143, 014015 (2011).

18. W. Vogel and J. Sperling, Phys. Rev. A 89, 052302 (2014).

19. C. T. Lee, Phys. Rev. A 44, R2775 (1991); N. Lükenhaus and S. M. Barnett, Phys. Rev. A 51, 3340 (1995); A. F. de Lima and B. Baseia, Phys. Rev. A 54, 4589 (1996); J. Janszky, M. G. Kim, and M. S. Kim, Phys. Rev. A 53, 502 (1996).

20. C. T. Lee, Phys. Rev. A 52, 3374 (1995). 
Informational 5th page.

\section{REFERENCES}

1. J. S. Bell, "On the Einstein Podolsky Rosen paradox," Physics 1, 195200 (1964); J. S. Bell, "On the Problem of Hidden Variables in Quantum Mechanics," Rev. Mod. Phys. 38, 447-452 (1966); J. F. Clauser and M. A. Horne, "Experimental consequences of objective local theories," Phys. Rev. D 10, 526-535 (1974); A. Fine, "Hidden Variables, Joint Probability, and the Bell Inequalities," Phys. Rev. Lett. 48, 291-295 (1982); I. Pitowsky, "George Boole's Conditions of Possible Experience and the Quantum Puzzle," Brit. J. Phil. Sci. 45, 95-125 (1994); T. Durt, "Three interpretations of the violations of Bell's inequalities," Found. Phys. 27, 415-434 (1997); A. Rivas, "Incompatible Statistics and BellKS Theorem," http://arxiv.org/abs/1501.04929.

2. W. M. Muynck, Foundations of Quantum Mechanics, an Empiricist Approach, (Kluwer Academic Publishers, 2002); W. M. de Muynck and $\mathrm{H}$. Martens, "Neutron interferometry and the joint measurement of incompatible observables," Phys. Rev. A 42, 5079-5085 (1990); W. M. de Muynck, "Information in neutron interference experiments," Phys. Lett. A 182, 201-206 (1993); W. M. de Muynck, "An alternative to the Lüuders generalization of the von Neumann projection, and its interpretation," J. Phys. A: Math. Gen. 31, 431-444 (1998).

3. H. S. Karthik, H. Katiyar, A. Shukla, T. S. Mahesh, A. R. Usha Devi, and A. K. Rajagopal, "Inversion of moments to retrieve joint probabilities in quantum sequential measurements," Phys. Rev. A 87, 052118 (2013).

4. L. Mandel and E. Wolf, Optical Coherence and Quantum Optics (Cambridge University Press, 1995); M. O. Scully and M. S. Zubairy, Quantum Optics (Cambridge University Press, 1997); D.-G. Welsch, W. Vogel, and T. Opatrný, "Homodyne detection and quantum-state reconstruction," in Progress in Optics, E. Wolf ed. (Elsevier Science, 1999) 39, 63-211.

5. A. Luis, "Effect of fluctuation measures on the uncertainty relations between two observables: Different measures lead to opposite conclusions," Phys. Rev. A 84, 034101 (2011); A. Luis, "Contradictory entropic joint uncertainty relations for complementary observables in two-level systems," http://arxiv.org/abs/1306.5211; A. Luis, G. M. Bosyk, and M. Portesi, "Entropic measures of joint uncertainty: effects of lack of majorization," Physica A 444, 905 (2016).

6. A. Luis, "Nonclassical states from the joint statistics of simultaneous measurements," http://arxiv.org/abs/1506.07680.

7. F. T. Arecchi, E. Courtens, R. Gilmore, and H. Thomas, "Atomic Coherent States in Quantum Optics," Phys. Rev. A 6, 2211-2237 (1972); O. Giraud, P. Braun, and D. Braun, "Classicality of spin states," Phys. Rev. A 78, 042112 (2008); O. Giraud, P. Braun, and D. Braun, "Quantifying quantumness and the quest for Queens of Quantum," New J. Phys. 12, 063005 (2010).

8. D. Arsenović, N. Burić, and D. M. Davidović, "Classical-like states of spin $s=1 / 2$," Phys. Rev. A 60, 1797-1801 (1999); A. Luis, "Nonclassical polarization states," Phys. Rev. A 73, 063806 (1-10) (2006); M. V. Chokhova, and F. Y. Khalili, "Nonclassical features of the polarization quasiprobability distribution," Phys. Rev. A 88, 023822 (1-10) (2013).

9. P. Carruthers and M. M. Nieto, "Phase and Angle Variables in Quantum Mechanics," Rev. Mod. Phys. 40, 411-440 (1968); S. M. Barnett and D. T. Pegg, "Phase in quantum optics," J. Phys. A 19, 3849-3862 (1986); R. Lynch, "Phase fluctuations in a squeezed state using measured phase operators," J. Opt. Soc. Am. B 4, 1723-1726 (1987); J. W. Noh, A. Fougères and L. Mandel, "Measurement of the quantum phase by photon counting," Phys. Rev. Lett. 67, 1426-1429 (1991); J. W. Noh, A. Fougères and L. Mandel, "Operational approach to the phase of a quantum field," Phys. Rev. A 45, 424-442 (1992); J. W. Noh, A. Fougères and L. Mandel, "Further investigations of the operationally defined quantum phase," Phys. Rev. A 46, 2840-2852 (1992); A. S. Shumovsky, "Quantum phase via the angular momentum," Opt. Commun. 136, 219-222 (1997); A. Cives-Esclop, A. Luis, and L. L. Sánchez-Soto, "Unbalanced homodyne detection with a weak local oscillator," Opt. Commun. 175, 153-161 (2000).

10. M. Grabowski, "Spin phase," Int. J. Theor. Phys. 28, 1215-1227 (1989); M. Grabowski, "On the phase operator," Rep. Math. Phys. 29, 377-
382 (1991); J. Bergou and B.-G. Englert, "Operators of the phase. Fundamentals," Ann. Phys. (N. Y.) 209, 479-505 (1991); R. Lynch, "The quantum phase problem: a critical review," Phys. Rep. 256, 367436 (1995); V. Peřinová, A. Lukš and J. Peřina, Phase in Optics (World Scientific, 1998).

11. A. Luis and L. L. Sánchez-Soto, "Quantum phase difference, phase measurements, and Stokes operators," in Progress in Optics, E. Wolf ed. (Elsevier, 2000) 41, 421-482 (2000); A. Luis and L. L. SánchezSoto, "Phase-difference operator," Phys. Rev. A 48, 4702-4708 (1993).

12. J. Vaccaro, "Number-phase Wigner function on Fock space," Phys. Rev. A 52, 3474-3488 (1995); A. Luis, "Nonclassical polarization states," Phys. Rev. A 73, 063806 (2006).

13. T. Kiss, U. Herzog, and U. Leonhardt, "Compensation of losses in photodetection and in quantum-state measurements," Phys. Rev. A 52, 2433-2435 (1995).

14. K. Vogel and H. Risken, "Determination of quasiprobability distributions in terms of probability distributions for the rotated quadrature phase," Phys. Rev. A 40, 2847-2849 (1989); A. I. Lvovsky, H. Hansen, T. Aichele, O. Benson, J. Mlynek, and S. Schiller, "Quantum State Reconstruction of the Single-Photon Fock State," Phys. Rev. Lett. 87, 050402 (2001).

15. N. G. Walker, "Quantum theory of multiport optical homodyning," J. Mod. Opt. 34, 15-60 (1987); O. Jedrkiewicz, R. Loudon, and J. Jeffers, "Retrodiction for coherent communication with homodyne or heterodyne detection," Eur. Phys. J. D 39, 129-140 (2006).

16. In passing, this recalls the Goethe formulation of vision as the meeting of the inner and outer lights at the eye, as recalled by A. G. Zajonc, "Goethe's Theory of Color and Scientific Intuition," Am. J. Phys. 44, 327-333 (1976).

17. A. Rivas and A. Luis, "Nonclassicality of states and measurements by breaking classical bounds on statistics," Phys. Rev. A 79, 042105 (2009); A. Luis and A. Rivas, "Independent nonclassical tests for states and measurements in the same experiment," Phys. Scr. T143, 014015 (2011).

18. W. Vogel and J. Sperling, "Unified quantification of nonclassicality and entanglement," Phys. Rev. A 89, 052302 (2014).

19. C. T. Lee, "Measure of the nonclassicality of nonclassical states," Phys. Rev. A 44, R2775-R2778 (1991); N. Lükenhaus and S. M. Barnett, "Nonclassical effects in phase space," Phys. Rev. A 51, 3340-3342 (1995); A. F. de Lima and B. Baseia, "Comment on Theorem on nonclassical states," Phys. Rev. A 54, 4589-4590 (1996); J. Janszky, M. G. Kim, and M. S. Kim, "Quasiprobabilities and the nonclassicality of fields," Phys. Rev. A 53, 502-506 (1996).

20. C. T. Lee, "Theorem on nonclassical states," Phys. Rev. A 52, 33743376 (1995). 